\title{
ANALYSIS OF POLE COORDINATE DATA PREDICTIONS IN THE EARTH ORIENTATION PARAMETERS COMBINATION OF PREDICTION PILOT PROJECT
}

\author{
W. Kosek ${ }^{1,2}$, B. Luzum ${ }^{3}$, M. Kalarus ${ }^{1}$, A. Wnęk ${ }^{2}$, M. Zbylut ${ }^{2}$ \\ ${ }^{1}$ Space Research Centre, Polish Academy of Sciences, Warsaw, Poland \\ ${ }^{2}$ Environmental Engineering and Land Surveying, University of Agriculture in Krakow, \\ Poland \\ ${ }^{3}$ U.S. Naval Observatory, Washington DC, USA \\ e-mail:kosek@cbk.waw.pl
}

\begin{abstract}
In October 2010 the US Naval Observatory together with the Space Research Centre in Warsaw initiated the Earth Orientation Parameters Combination of Prediction Pilot Project, which was accepted by the IERS Directing Board. The goal of this project is to determine the feasibility of combining Earth Orientation Parameters (EOP) predictions on an operational basis. The ensemble predictions of EOPs are more accurate than the results from individual predictions. The pole coordinate data predictions from different prediction contributors and ensemble predictions computed by the U.S. Naval Observatory are studied to determine the statistical properties of polar motion forecasts by looking at second, third and fourth moments about the mean. The increase of prediction errors in pole coordinate data can be due to the change of phase of the annual oscillation in the joint atmospheric-ocean excitation function.
\end{abstract}

Keywords: Earth rotation, prediction.

\section{INTRODUCTION}

The International Celestial and Terrestrial Reference Systems (ITRS and ICRS) are defined by coordinates of extragalactic radio sources observed by VLBI and by the coordinates and velocities of sites, respectively, determined from space geodetic techniques e.g. GNSS, DORIS (Doppler Orbitography and Radiopositioning Integrated by Satellite), SLR, LLR and VLBI. The EOP predictions enable the real time transformation between the realizations of these systems that are the International Celestial and Terrestrial Reference Frames (ICRF and ITRF). During the last decades the definition and practical realization of these systems has been adapting to increasing accuracy of the measurements provided by these techniques. The real time transformation between these two frames is a function of time realized by predictions of $\mathrm{x}, \mathrm{y}$ pole coordinates, UT1-UTC and a precesion-nutation extrapolation model together with the predictions of $d X, d Y$ precesion-nutation residuals. The 
determination accuracy of these EOPs observed by space geodetic techniques is of the order of few millimeters on the Earth's surface, however such accuracy is not possible in the real time transformation due to the latency of products provided by these techniques. Thus, it is necessary to predict these transformation parameters. However their prediction error even for a few days in the future is several times less than their determination accuracy (Kosek at al. 2004, Luzum 2010). Predictions of EOPs are now provided by the IERS Rapid Service Prediction Centre in USNO, Washington DC. The $\mathrm{x}$, y pole coordinates are predicted using a combination of LS extrapolation and autoregressive prediction of the least-squares (LS) residuals (Kosek et al. 2004). The short-term UT1-UTC data are predicted using the forecast of the axial component of atmospheric angular momentum obtained during the estimation of a global dynamic atmospheric circulation model (Johnson et al. 2005). The accuracy of the new IAU2000 precession-nutation model is very high, thus its differences with respect to observations are very small and show a quasi-periodic signal related to the free core nutation (FCN) with an amplitude not exceeding 0.3 mas. The accuracy of the real time transformation between the celestial and terrestrial reference frames mostly depends on the prediction accuracy of the $\mathrm{x}$, y pole coordinates and UT1-UTC data.

The predictions of EOPs are being used by the NASA Deep Space Network (DSN) which consists of the network of antennas located in 1) Goldstone, California, Mojave desert; 2) near Madrid, Spain; and 3) near Canberra, Australia to control interplanetary missions, radio and radar astronomical observations as well as some Earth orbiting satellites. The longitude distance of about $120^{\circ}$ between these sites enables observations of space objects regardless of Earth rotation. High accuracy prediction of EOPs becomes very important for the realization of altimetric and gravimetric missions as well as the determination of precise directions of extragalactic radio sources observed by VLBI.

\section{INTERNATIONAL COOPERATION}

To improve the EOP prediction accuracy, international cooperation has become necessary due to degradation of the EOP prediction accuracy with prediction length. This prediction accuracy degradation is mostly caused by variable short period oscillations in $\mathrm{x}, \mathrm{y}$ pole coordinates and UT1-UTC data. Such wideband, short-period oscillations in pole coordinates and universal time data are excited by wideband high-frequency oscillations of equatorial components of the joint atmospheric-ocean excitation function and the axial component of atmospheric excitation function, respectively.

The first campaign, named Earth Orientation Parameters Prediction Comparison Campaign (EOPPCC), was initiated at the Technical University of Vienna by Prof. H. Schuh and Space Research Centre of Polish Academy of Sciences (SRC PAS) by W. Kosek and M. Kalarus. The goal of this campaign, which started in Oct. 2005 and terminated in Mar. 2008, was the comparison of the EOP prediction results computed by different prediction techniques using different input data. About 10 participants of this campaign computed EOP predictions every week that were then analyzed at the SRC PAS. The most important achievement of this campaign was to show that ensemble predictions of $\mathrm{x}$, $\mathrm{y}$ pole coordinate data are more accurate than the individual ones and the most accurate short-term prediction method for UT1-UTC was the Kalman filter which used the 10-day forecast of the axial component of atmospheric angular momentum as an input together with UT1-UTC data (Kalarus et al. 2010).

In Apr. 2006 at the EGU the IERS Working Group on Predictions (WGP) was initiated to study the advantages and shortfalls of different EOP prediction algorithms as well as to determine what prediction products are useful to the user community in addition to making a detailed examination of the fundamental properties of the different input data sets. Due to the initiative of the WGP members, in Oct. 2009 the IERS Workshop on Combination and 
Prediction was held in Warsaw. The goals of this Workshop were to determine the current state-of-the-art for the EOP prediction in terms of data sets and algorithms as well as to discuss recommended actions for improving EOP predictions, including improving the IERS rapid combined series. This Workshop generated about 20 recommendations related to observations, analysis and prediction of EOPs. Following these recommendations in Oct. 2010 the Earth Orientation Parameters Combination of Prediction Pilot Project (EOPCPPP), was initiated by the IERS Directing Board. The goal of this Project is to determine the feasibility and benefits of combining EOP predictions on a daily basis and to determine the best algorithms for EOP prediction combinations (USNO 2011, SRC 2011). In this Project about 10 participants provide EOP predictions ranging from roughly 90 days up to one year (Tab. 1).

Table 1. The participants of the EOPCPPP and their contribution. See http://maia.usno.navy.mil/eopcppp/ or http://www.cbk.waw.pl/eopcppp for additional details.

\begin{tabular}{|c|c|c|c|c|c|c|}
\hline Author & Institute & $\mathrm{x}, \mathrm{y}$ & $\begin{array}{c}\text { UT1 } \\
- \\
\text { UTC }\end{array}$ & $\Delta$ & $\mathrm{dX}, \mathrm{dY}$ & References \\
\hline \multicolumn{7}{|c|}{ Provided EOP predictions } \\
\hline $\begin{array}{l}\text { Brian } \\
\text { Luzum } \\
(B L)\end{array}$ & $\begin{array}{l}\text { U.S. Naval Observatory, } \\
\text { Washington DC, USA }\end{array}$ & + & + & - & - & Luzum et al. 2010 \\
\hline $\begin{array}{l}\text { Daniel } \\
\text { Gambis } \\
\text { (DG) }\end{array}$ & $\begin{array}{l}\text { Paris Observatory, Paris, } \\
\text { France }\end{array}$ & + & + & + & + & $\begin{array}{c}\text { Gambis 2004; Gambis et al. } \\
\text { 2008, 2010; Bizourad and } \\
\text { Gambis } 2009\end{array}$ \\
\hline $\begin{array}{l}\text { Leonid } \\
\text { Zotov } \\
(\text { LZ) }\end{array}$ & $\begin{array}{l}\text { Sternberg Astronomical } \\
\text { Institute of Moscow State } \\
\text { University, Department of } \\
\text { Gravimetry, Moscow, Russia }\end{array}$ & + & + & + & - & Zotov 2010 \\
\hline $\begin{array}{l}\text { Maciej } \\
\text { Kalarus } \\
\text { (MK) }\end{array}$ & $\begin{array}{l}\text { Space Research Centre, PAS, } \\
\text { Warsaw, Poland }\end{array}$ & + & - & - & - & Kalarus et al. 2008, 2010 \\
\hline $\begin{array}{l}\text { Richard } \\
\text { Gross } \\
\text { (RG) }\end{array}$ & $\begin{array}{l}\text { Jet Propulsion Laboratory, } \\
\text { Pasadena, California, USA }\end{array}$ & + & + & + & - & $\begin{array}{l}\text { Gross 2005; Gross et al. } \\
\text { 1998; Friedman et al. 1994; } \\
\text { Morabito et al. } 1998\end{array}$ \\
\hline $\begin{array}{l}\text { Viktor } \\
\text { Tissen } \\
\text { (VT) }\end{array}$ & $\begin{array}{l}\text { Siberian Scientific Research } \\
\text { Institute of Metrology and } \\
\text { Siberian State Geodetic } \\
\text { Academy, Russia } \\
\end{array}$ & + & + & - & - & Tissen et al. 2009, 2010 \\
\hline $\begin{array}{l}\text { Wiesław } \\
\text { Kosek } \\
\text { (WK) }\end{array}$ & $\begin{array}{l}\text { Space Research Centre, PAS, } \\
\text { Warsaw, Poland }\end{array}$ & + & - & - & - & Kosek et al. 2004 \\
\hline $\begin{array}{l}\text { Xu } \\
\text { Xueqing } \\
\text { (XX) }\end{array}$ & $\begin{array}{l}\text { Shanghai Astronomical } \\
\text { Observatory, China }\end{array}$ & + & + & + & - & \\
\hline $\begin{array}{l}\text { Zinovy } \\
\text { Malkin } \\
(\mathrm{ZM})\end{array}$ & Pulkovo Observatory, Russia & + & + & + & + & $\begin{array}{l}\text { Malkin Z., Skurikhina 1996; } \\
\text { Malkin 2007, 2010a,b }\end{array}$ \\
\hline \multicolumn{7}{|c|}{ Provided EOP ensemble predictions } \\
\hline $\begin{array}{l}\text { Brian } \\
\text { Luzum } \\
\text { (BL-ep) }\end{array}$ & $\begin{array}{l}\text { U.S. Naval Observatory, } \\
\text { Washington DC, USA }\end{array}$ & + & + & + & + & \\
\hline
\end{tabular}




\section{ANALYSIS}

To analyze prediction results of the individual participants of the project as well as ensemble predictions computed daily at the US Naval Observatory the following statistics were applied: standard deviation (SD), mean absolute error (MEA), skewness (SKE) and kurtosis (KUR). The prediction accuracy can be expressed using the first two statistics: SD and MAE. The SD of predictions computed by the individual participant or for the ensemble prediction for the $i^{t h}$ day in the future is computed by the following formula:

$$
S D_{i}=\sqrt{1 / n_{p} \sum_{j=1}^{n_{p}}\left(x_{i, j}^{p r e d}-x^{o b s}-\mu_{i, j}\right)^{2}}, \quad i=1,2, \ldots, M
$$

where $\mu_{i, j}=1 / n_{p} \sum_{j=1}^{n_{p}}\left(x_{i, j}^{\text {pred }}-x^{\text {obs }}\right), \quad i=1,2, \ldots M$, is the first moment about the mean, $x_{i, j}^{\text {pred }}$ is the $j^{\text {th }}$ prediction computed for the $i^{\text {th }}$ day in the future, $x^{\text {obs }}$ is the observed future value of time series, $M$ is the maximum prediction length, $n_{p}$ is the number of predictions. formula:

The statistical error of SD for the $i^{\text {th }}$ day in the future is computed by the following

$$
\hat{\sigma}(S D)_{i}=S D_{i} / \sqrt{2 n_{p}}
$$

The MAE of predictions for the $i^{\text {th }}$ day in the future is computed by the following formula:

$$
M A E_{i}=1 / n_{p} \sum_{j=1}^{n_{p}}\left|x_{i, j}^{p r e d}-x^{o b s}\right|, \quad i=1,2, \ldots, M
$$

The statistical error of MAE for the $i^{\text {th }}$ day in the future is computed by the following formula (Kalarus et al. 2010):

$$
\hat{\sigma}(M A E)_{i}=S D_{i} \sqrt{(\pi-2) /\left(\pi \cdot n_{p}\right)}
$$

Skewness (SKE) is a measure of the asymmetry of the probability distribution of a realvalued random variable. Negative skewness indicates that the tail on the left side of the probability density function is longer than the right side. If the distribution is symmetric then skewness is equal to zero. The SKE of predictions for the $i^{\text {th }}$ day in the future are computed by the following formula:

$$
S K E_{i}=E\left[\left(\frac{x_{i, j}^{\text {pred }}-x^{o b s}-\mu_{i, j}}{S D_{i}}\right)^{3}\right]=\frac{1 / n_{p} \sum_{i=1}^{n_{p}}\left(x_{i, j}^{\text {pred }}-x^{o b s}-\mu_{i, j}\right)^{3}}{\left[1 / n_{p} \sum_{i=1}^{n_{p}}\left(x_{i, j}^{\text {pred }}-x^{o b s}-\mu_{i, j}\right)^{2}\right]^{3 / 2}}=\frac{\mu_{3}}{S D_{i}^{3}}, i=1,2, \ldots, M
$$

The statistical error of SKE for the $i^{\text {th }}$ day in the future depends only on the number of predictions and is computed by the following formula:

$$
\hat{\sigma}(S K E)_{i}=\sqrt{6 / n_{p}} .
$$


Kurtosis (KUR) or excess kurtosis is a measure of the "peakedness" of the probability distribution of a real-valued random variable. If kurtosis is equal to 0 then the differences between pole coordinate data and their predictions follow a normal distribution. If it is negative then the probability distribution is more flat which means that there are a larger number of extreme deviations in the variance and if its positive then the number of large deviations in the variance is less than in the case of normal distribution. The kurtosis of predictions for the $i^{\text {th }}$ day in the future are computed by the following formula:

$K U R_{i}=E\left[\left(\frac{x_{i, j}^{\text {pred }}-x^{o b s}-\mu_{i, j}}{S D_{i}}\right)^{4}\right]-3=\frac{1 / n_{p} \sum_{i=1}^{n_{p}}\left(x_{i, j}^{\text {pred }}-x^{o b s}-\mu_{i, j}\right)^{4}}{\left.1 / n_{p} \sum_{i=1}^{n_{p}}\left(x_{i, j}^{\text {pred }}-x^{o b s}-\mu_{i, j}\right)^{2}\right]^{2}}-3=\frac{\mu_{4}}{S D_{i}^{4}}-3$.

The statistical error of KUR for the $i^{\text {th }}$ day in the future is computed by the following formula:

$$
\hat{\sigma}(K U R)_{i}=\sqrt{24 / n_{p}} \text {. }
$$

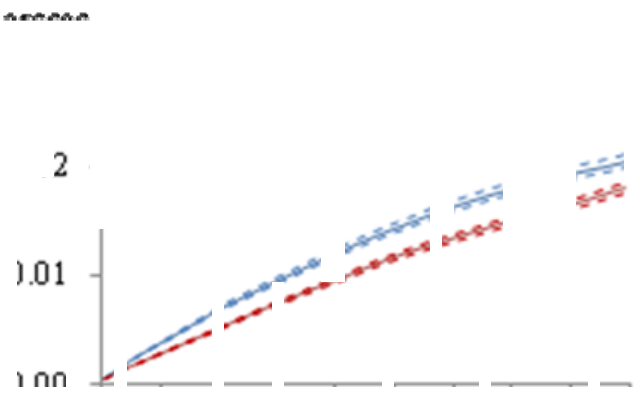

areser:

[.]2
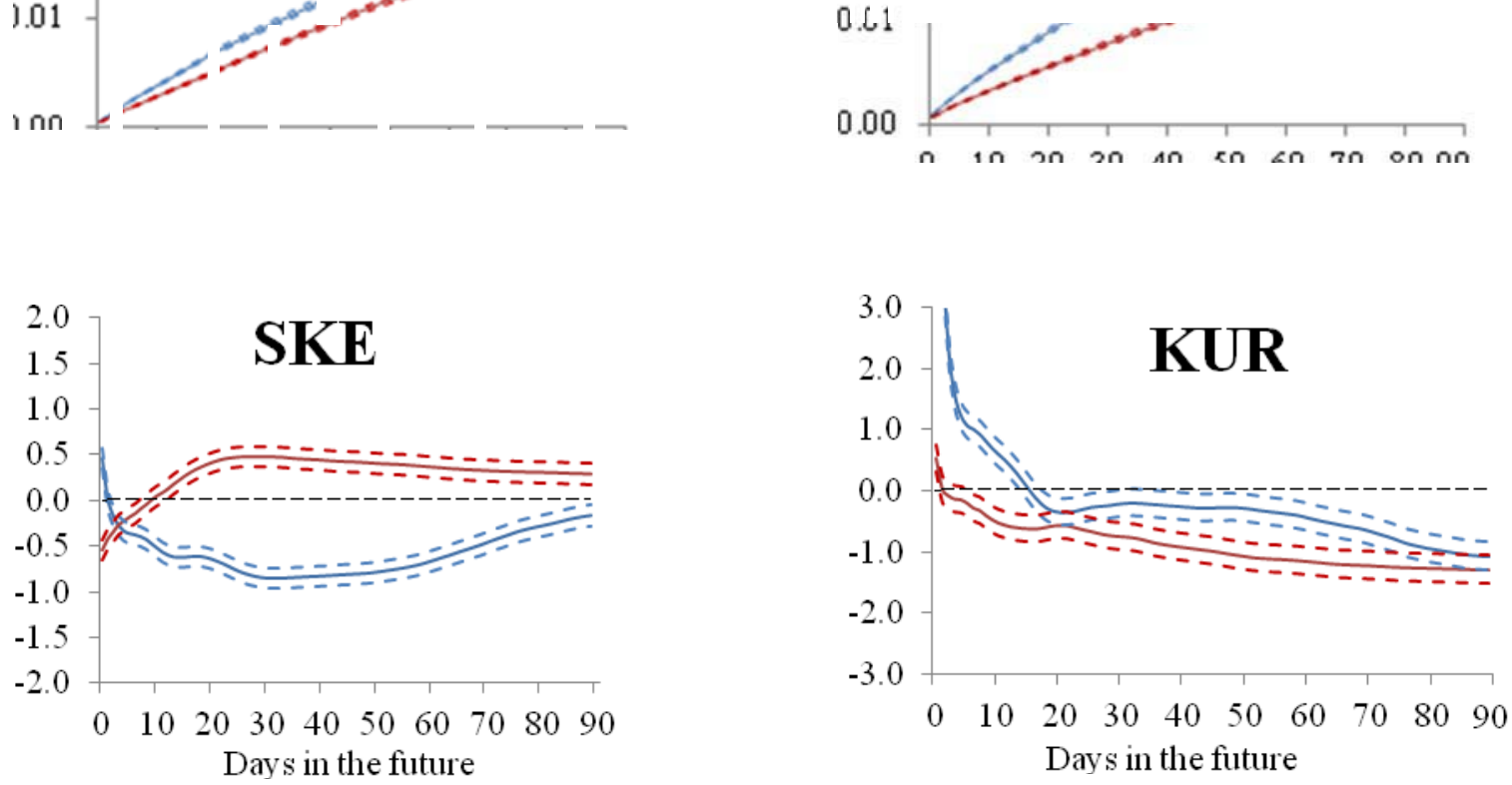

Fig. 1. Mean absolute error, standard deviation, skewness and kurtosis together with their error bars of $\mathrm{x}$ (blue), y (red) predictions computed by WK. 
arcsec

C. 0.2

C. 11

0.00
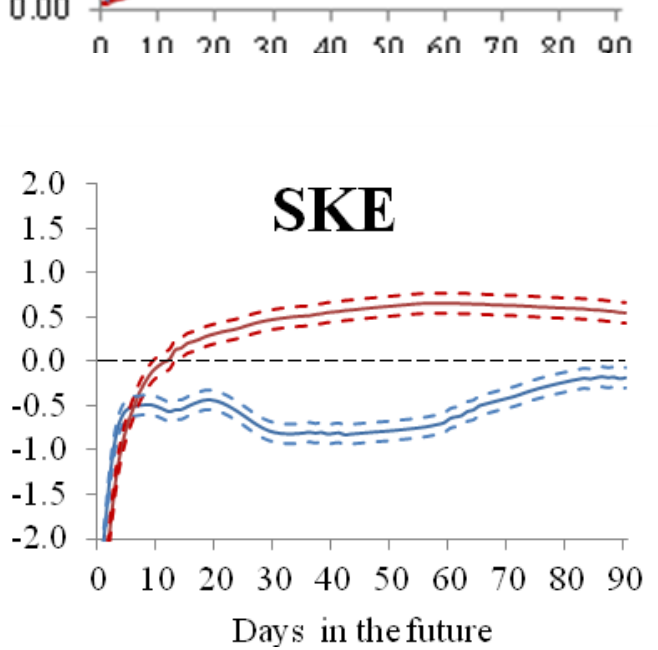

arcear
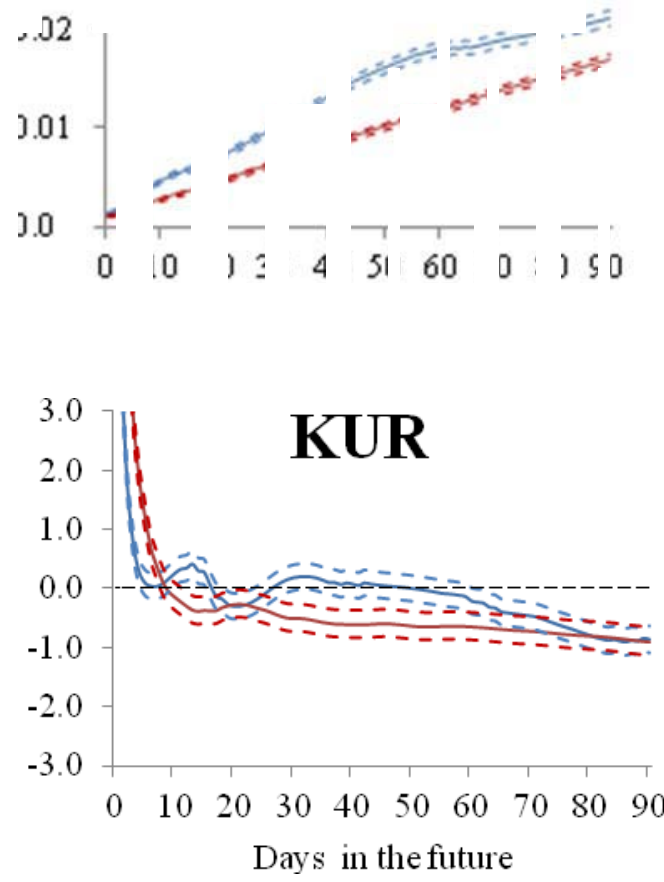

Fig. 2. Mean absolute error, standard deviation, skewness and kurtosis together with their error bars of $\mathrm{x}$ (blue), $\mathrm{y}$ (red) predictions computed by BL.

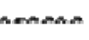

2
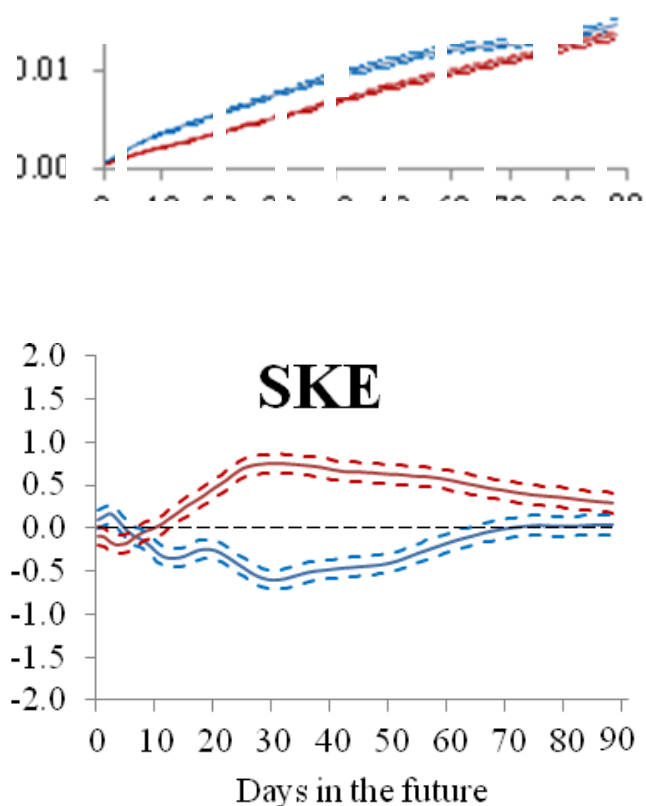

$(1,0$

(1). 1.

0.00
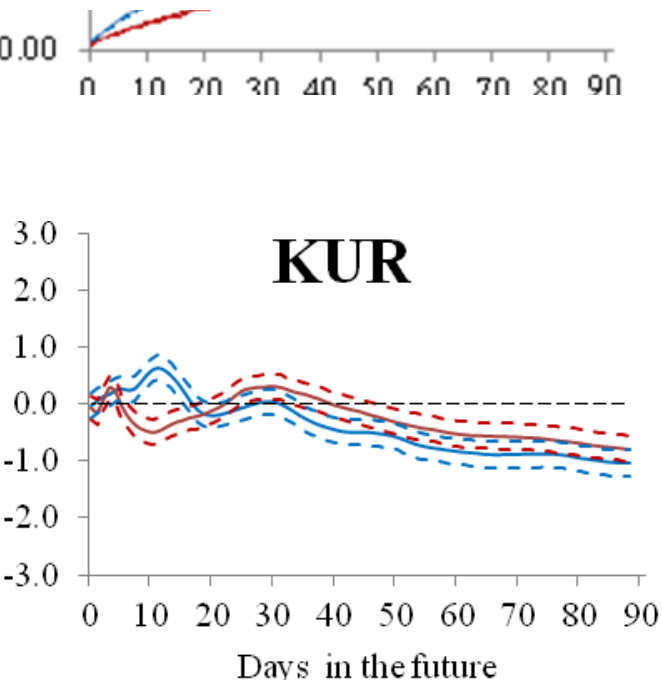

Fig. 3. Mean absolute error, standard deviation, skewness and kurtosis together with their error bars of x (blue), y (red) predictions computed by VT. 

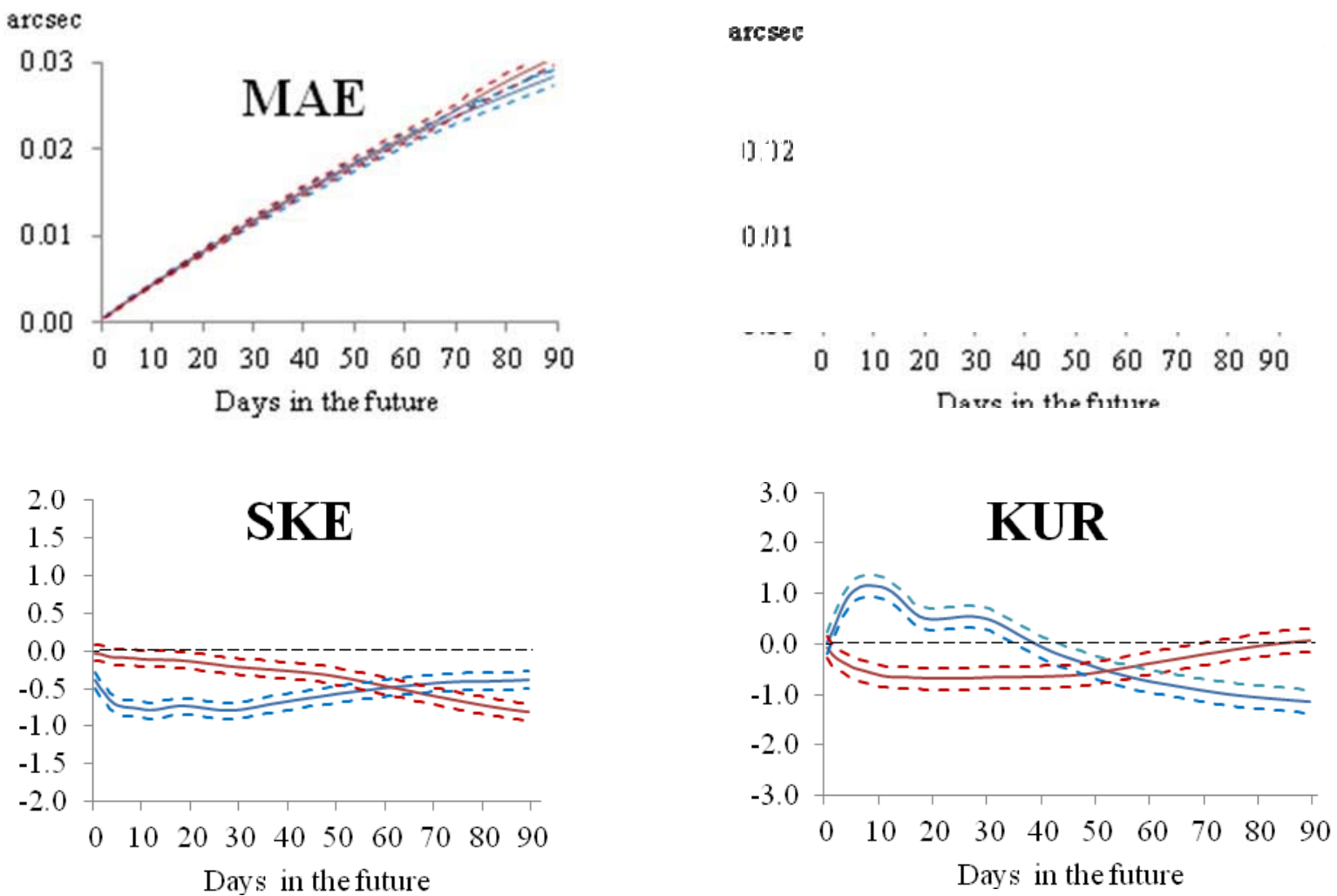

Fig. 4. Mean absolute error, standard deviation, skewness and kurtosis together with their error bars of $\mathrm{x}$ (blue), y (red) predictions computed by ZM.
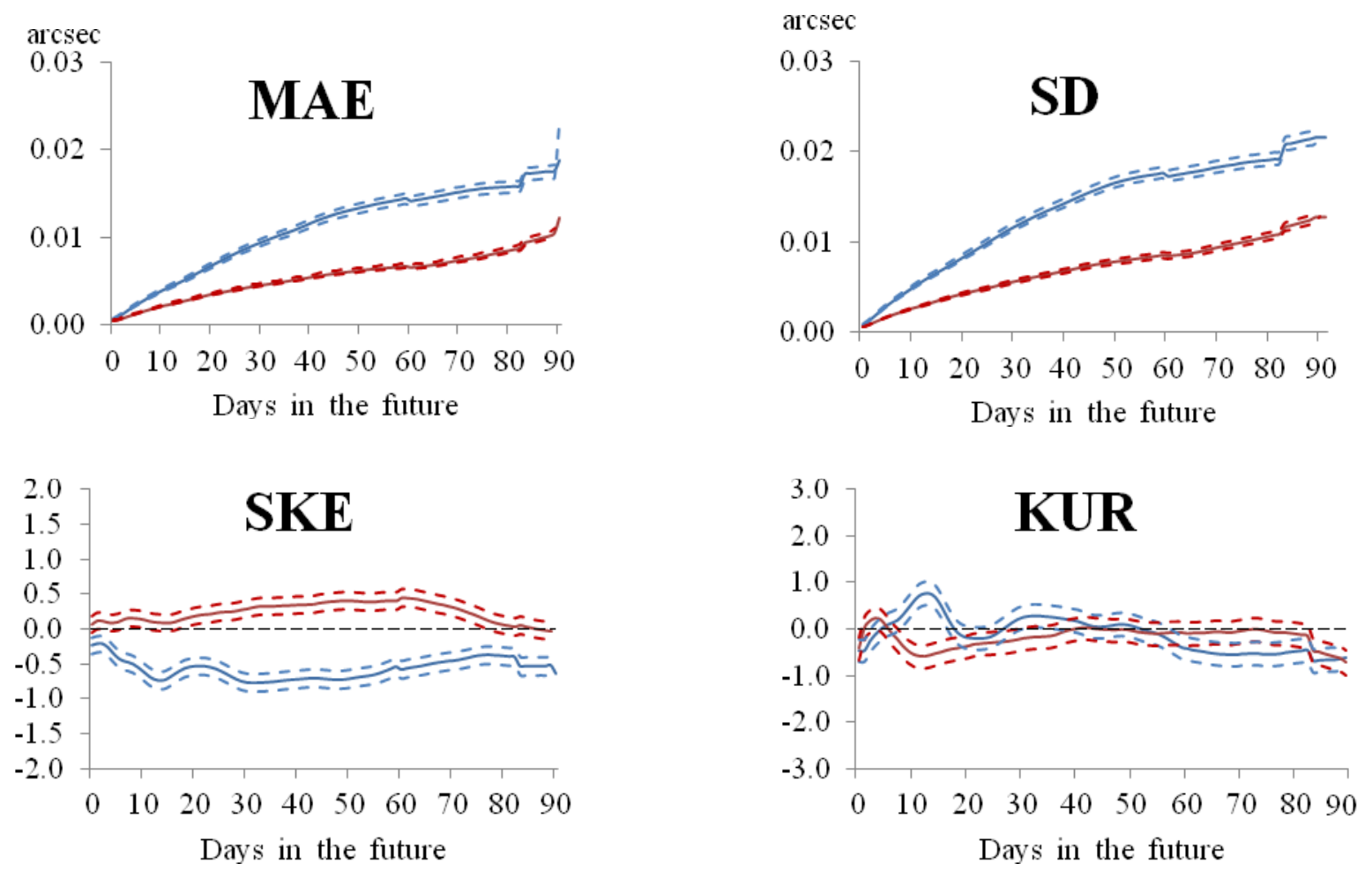

Fig. 5. Mean absolute error, standard deviation, skewness and kurtosis together with their error bars of $\mathrm{x}$ (blue), y (red) ensemble predictions computed by BL-ep. 
All four statistics were computed for $\mathrm{x}$, $\mathrm{y}$ pole coordinate data predictions provided by BL, WK, VT and ZM as well as ensemble predictions of these data provided by BL-ep. Figures 1-5 show the values of SD, MAE, SKE and KUR as a function of prediction length for chosen participants of the project. It can be seen that for different participants/prediction methods the skewness changes are similar. The negative value of skewness for the $\mathrm{x}$ pole coordinate is associated with a positive value for the y pole coordinate except for participant ZM where the skewness values are negative (Fig. 4). Negative or positive values of skewness suggest that the probability distribution of the differences between the pole coordinate data and their predictions are not symmetrical. A similar change of skewness suggests also that there are irregular variations in the pole coordinate data that are difficult to predict by any method.

The kurtosis values for different participants are usually similar and decrease with the prediction length except the y pole coordinate of participant ZM (Fig. 4). Their values are usually greater for the $\mathrm{x}$ pole coordinate than for $\mathrm{y}$ except participant VT (Fig. 3) and ensemble predictions (Fig. 5) where they are of the same order. A decrease of kurtosis with prediction length suggests that the probability distribution becomes more flat and has larger tails than a normal distribution.
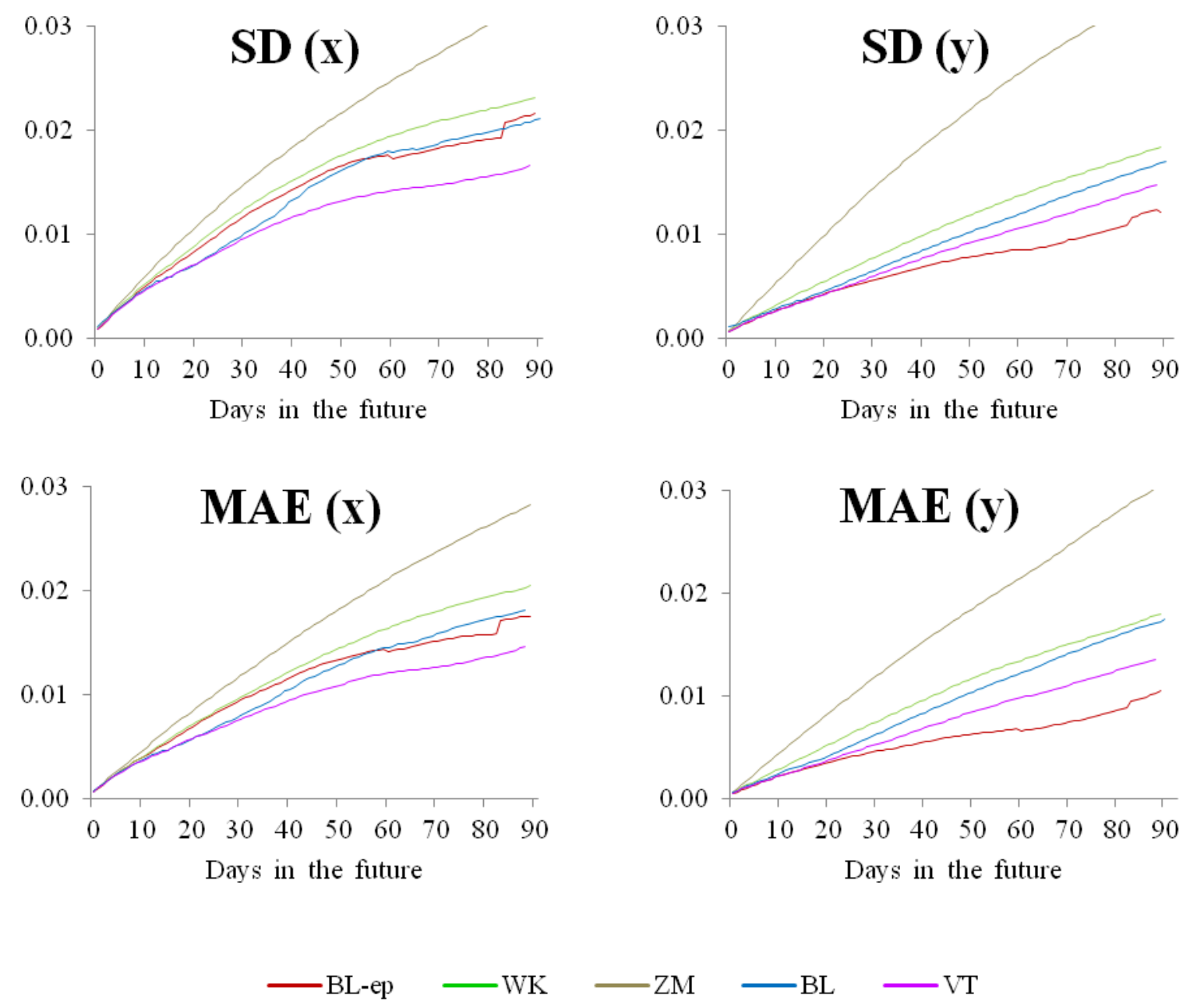

Fig. 6. Standard deviations and mean absolute errors computed by different participants of the project and of ensemble predictions computed by USNO (BL-ep) (red). 
Figure 6 shows the mean prediction errors of pole coordinate data represented by the standard deviations and mean absolute errors computed for different participants of the project and of ensemble predictions computed by USNO. Note that the mean prediction errors of the ensemble prediction are greater than the smallest mean prediction errors for an individual participant for the x pole coordinate. The mean ensemble prediction error of the $y$ pole coordinate for prediction lengths less than 20 days is of the order of the smallest prediction error for an individual participant.

To explain the skewness variations for different participants, the differences between pole coordinate data and their least-squares + autoregressive (LS+AR) predictions (Kosek et al. 2004) of WK were computed (Fig. 7). In this prediction method the IERS eopc04.62-now data were used (IERS 2011). The skewness for the x pole coordinate predictions is usually less than for $\mathrm{y}$ (Figs. 1-5) and these differences show that the sign for $\mathrm{x}$ is mostly negative while the sign for $y$ is mostly positive during the EOPCPPP. Note that between the EOPPCC and the EOPCPPP the sign of these differences for the $\mathrm{x}$ and $\mathrm{y}$ pole coordinate is mostly negative which indicates that the tail on the left side of the probability density function is longer than the right side, and it deviates significantly from a normal distribution. Note that between 2004 and 2009 the change of sign of these differences for the y pole coordinate shows a quasi-annual variation. Because the last 10 years of pole coordinate data is used in the least-squares model of the LS+AR prediction (Kosek et al. 2004), the constant phase of the annual oscillation in the extrapolation model represents the average phase for this time span of data. This constant phase in the extrapolation model is not equal to its real value for the prediction epoch due to significant phase variations of the annual oscillation in pole coordinate data (Kosek et al. 2006).

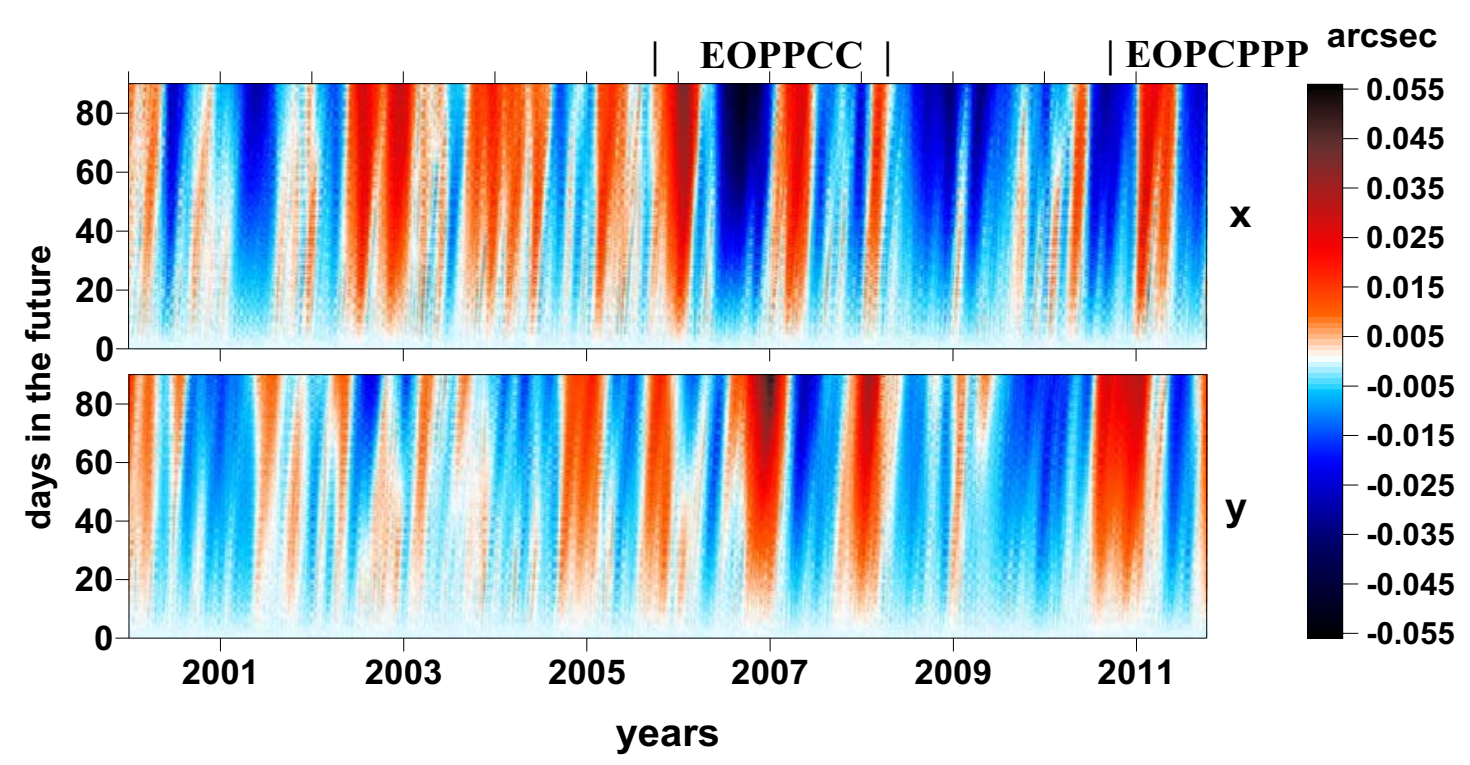

Fig. 7. The differences between IERS pole coordinate data and their LS + AR predictions computed by WK for 90 days in the future.

It was found that the short term (up to 100 days in the future) mean prediction errors of pole coordinate data are of the same order as the mean prediction errors of the pole coordinate model data computed from joint atmospheric-ocean excitation functions (Kosek 2010, Kosek et al. 2011). The semblance function (Kosek et al. 2011) and polarization function (Kosek 2010) of pole coordinate data and pole coordinate model data computed from the joint atmospheric-ocean excitation functions are very similar especially for the annual frequency 
band which means that the annual oscillation in pole coordinate data is mostly excited by the joint atmospheric-ocean excitation function. Thus, the main cause of short-term prediction errors of pole coordinate data can be due to irregular phase variations of the annual oscillation in the atmospheric-ocean excitation. The autoregressive prediction of the least-squares extrapolation residuals was not able to take these phase variations of the annual oscillation into account.

\section{CONCLUSIONS}

The mean ensemble prediction error of the $\mathrm{x}$ pole coordinate is larger than the smallest prediction error for an individual participant and the mean ensemble prediction error of the $y$ pole coordinate for prediction lengths less than 20 days is of the order of the smallest prediction error for individual participant.

The skewness values for different participants show that the probability distribution becomes more nonsymmetrical when the prediction length increases. Usually, during the EOPCPPP the signs of skewness are opposite for $\mathrm{x}$ and $\mathrm{y}$ pole coordinate predictions.

The kurtosis values usually decrease with the prediction length, which means that the probability distribution becomes more flat and has larger tails than a normal distribution.

The increase of short-term prediction errors of pole coordinate data can be caused by irregular variations of the annual oscillation phase in polar motion and its atmospheric-ocean excitation.

\section{REFERENCES}

Bizouard C and D. Gambis, 2009, The combined solution C04 for Earth Orientation Parameters, recent improvements, Springer Verlag series, Series International Association of Geodesy Symposia, Vol. 134 Drewes, Hermann (Ed.), 265-270.

Freedman, A. P., J. A. Steppe, J. O. Dickey, T. M. Eubanks, and L.-Y. Sung, 1994, The shortterm prediction of universal time and length of day using atmospheric angular momentum, J. Geophys. Res., 99, 6981-6996.

Gambis D., 2004, Monitoring Earth orientation using space-geodetic techniques: state-of-theart and prospective, J. of Geodesy, Volume 78, Issue 4-5, pp. 295-303, DOI 10.1007/s00190-004-0394-1

Gambis D., J.Y. Richard, D. Salstein, 2008, Use of Atmospheric Angular Momentum forecasts for UT1 prediction, Proc. Journées Systèmes de Référence 2007, N. Capitaine (ed.), 210-212.

Gambis, D., Salstein D., Lambert S., 2010, Use of Atmospheric Angular momentum forecasts for UT1 predictions, Subm. to JoG.

Gross, R. S., 2005, Combinations of Earth orientation measurements: SPACE2004, COMB2004, and POLE2004, Jet Propulsion Laboratory Publ. 05-6, 28 pp., Pasadena, Calif., 2005.

Gross, R. S., T. M. Eubanks, J. A. Steppe, A. P. Freedman, J. O. Dickey, and T. F. Runge, A., 1998, Kalman filter-based approach to combining independent Earth orientation series, J. Geodesy, 72, 215-235.

IERS 2011, http://hpiers.obspm.fr/iers/eop/eopc04/eopc04.62-now 
Johnson T., Luzum B.J. and Ray J.R., 2005, Improved near-term Earth rotation predictions using atmospheric angular momentum analysis and forecasts, Journal of Geodynamics, 39, 209-221.

Kalarus M., Kosek W., Schuh H., 2008, Current results of the Earth Orientation Parameters Prediction Comparison Campaign, Proc. Journées 2007, Systèmes de Référence SpatioTemporels "The Celestial Reference Frame for the Future". N. Capitaine (eds.), Observatoire de Paris Systèmes de Référence Temps-Espace UMR8630/CNRS, Paris, FRANCE, p. 159-162.

Kalarus M., Schuh H., Kosek W., Akyilmaz O., Bizouard Ch., Gambis D., Gross R., Jovanovic B., Kumakshev S., Kutterer H., Ma L., Mendes Cerveira P. J., Pasynok S., Zotov L., 2010, Achievements of the Earth Orientation Parameters Prediction Comparison Campaign, Journal of Geodesy (2010) 84: DOI: 10.1007/s00190-0100387-1, 587-596.

Kosek W., McCarthy D.D., Johnson T.J., Kalarus M., 2004, Comparison of polar motion prediction results supplied by the IERS Sub-bureau for Rapid Service and Predictions and results of other prediction methods. Proc. Journées 2003 Systèmes de Référence Spatio-Temporels, A. Finkelstein and N. Capitaine (eds.), pp. 164-169.

Kosek W., Rzeszótko A. and Popiński W., 2006, Phase variations of oscillations in the Earth orientation parameters detected by the wavelet technique. Proc. Journées 2005 Systèmes de Référence Spatio-Temporels. 121-124.

Kosek W., 2010, Causes of prediction errors of pole coordinates data, Proc. The 6th Orlov's Conference, "The study of the Earth as a planet by methods of geophysics, geodesy and astronomy ", Jun 22-24, 2009, MAO NAS of Ukraine, Kiev, Ukraine. (ed. Ya. Yatskiv) pp. 96-103.

Kosek W., Popiński W., Niedzielski T., 2011, Wavelet based comparison of high frequency oscillations in the geodetic and fluid excitation functions of polar motion, Proc. Journées 2010 Systèmes de référence spatio-temporels "New challenges for reference systems and numerical standards in astronomy" in. N. Capitaine (ed.), Observatoire de Paris, pp. 168-171.

Luzum B., 2010, Future of Earth Orientation Predictions, Artificial Satellites Vol. 45, No 2 / 2010, 10.2478/v10018-010-0011-X

Luzum, B., Stamatakos, N., Carter, M.S. Stetzler, B., and Shumate, N., 2010, "Rapid Service/Prediction Centre," in IERS Annual Report 2007, pp. 68-77.

Malkin Z. M. 2007, Empiric Models of the Earth's Free Core Nutation. Solar System Research, 2007, v. 41, No. 6, 492-497.

Malkin Z. M. 2010a, Analysis of the Accuracy of Prediction of the Celestial Pole Motion. Astronomy Reports, 2010, v. 54, No. 11, 1053-1061.

Malkin Z. 2010b, Employing combination procedures to short-time EOP prediction. Artificial Satellites, 2010, v. 45, No. 2, 87-93.

Malkin Z., Skurikhina E. 1996, On Prediction of EOP. Communications of IAA, 1996, No. 93.

Morabito, D. D., T. M. Eubanks, and J. A. Steppe, 1998, Kalman filtering of Earth orientation changes, in The Earth's Rotation and Reference Frames for Geodesy and Geodynamics, edited by A. K. Babcock and G. A. Wilkins, pp. 257-267, D. Reidel, Dordrecht, Holland, 1988. 
SRC 2011, http://www.cbk.waw.pl/eopcppp/cp.html

Tissen V. M., Tolstikov A. S., Balahnenko A. Yu., Malkin Z. M. 2009, High precision prediction of universal time based on 100-year data. Measurement Techniques, 2009, v. 52., No. 12, 1249-1255. DOI 10.1007/s11018-010-9429-6

Tissen V., Tolstikov A., Malkin Z. 2010, UT1 prediction based on long-time series analysis. Artificial Satellites, 2010, v. 45, No. 2, 111-118.

USNO 2011, http://maia.usno.navy.mil/eopcppp/contributions.html

Zotov L., 2010, Dynamical modeling and excitation reconstruction as fundamental of Earth rotation prediction, Artificial Satellites , Vol. 45, No 2 / 2010, 95-106.

Received: 2011-11-30,

Reviewed: 2012-02-27, by Z. Malkin,

Accepted: 2012-03-15. 